\title{
Minimax Approximate Solutions of Linear Boundary Value Problems
}

\author{
By Darrell Schmidt* and Kenneth L. Wiggins
}

Abstract. Define the operator $D: C^{\prime \prime}[0, \tau] \rightarrow C[0, \tau]$ by $D[u]=u^{\prime \prime}-a_{0} u^{\prime}-$ $a_{1} u$ where $a_{0}, a_{1} \in C[0, \tau]$ and consider the two point boundary value problem (BVP) $D[y](x)=a_{2}(x), x \in[0, \tau], N_{0}[y]=\alpha_{0} y(0)+\alpha_{1} y^{\prime}(0)=\alpha_{2}, N_{\tau}[y]=$ $\beta_{0} y(\tau)+\beta_{1} y^{\prime}(\tau)=\beta_{2}$ where $a_{2} \in C[0, \tau], \alpha_{0}^{2}+\alpha_{1}^{2} \neq 0$ and $\beta_{0}^{2}+\beta_{1}^{2} \neq 0$. Let $\Pi_{k}$ denote the set of polynomials of degree at most $k$ and define the approximating set $P_{k}=\left\{p \in \Pi_{k}: N_{0}[p]=\alpha_{2}, N_{\tau}[p]=\beta_{2}\right\}$. Then for each $k \geqslant 3$ there exists $p_{k} \in P_{k}$ satisfying $\left\|D\left[p_{k}\right]-a_{2}\right\|=\inf _{p \in P_{k}}\left\|D[p]-a_{2}\right\|=\delta_{k}$, where $\|\cdot\|$ denotes the uniform norm on $C[0, \tau]$. If the homogeneous BVP $D[y]=0, N_{0}[y]=$ $N_{\tau}[y]=0$ has no nontrivial solutions, then the nonhomogeneous BVP has a unique solution $y$ and $\lim _{k \rightarrow \infty}\left\|p_{k}^{(i)}-y^{(i)}\right\|=0$ for $i=0,1,2$. If $X$ denotes a closed subset of $[0, \tau]$ and

$$
\left.\delta_{k, X}=\inf _{p \in P_{k}} \max _{x \in X}|D| p\right](x)-a_{2}(x) \mid,
$$

then for each $\epsilon>0$ there exists $\delta>0$ such that $d(X) \leqslant \delta$ implies that $0 \leqslant \delta_{k}$ $\delta_{k, X} \leqslant \epsilon$, where $d(X)$ denotes the density of $X$ in $[0, \tau]$. Several numerical examples are given.

1. Introduction. Consider the linear two point boundary value problem

$$
\begin{aligned}
y^{\prime \prime}= & a_{0}(x) y^{\prime}+a_{1}(x) y+a_{2}(x), \quad x \in[0, \tau], \\
& N_{0}[y]=\alpha_{0} y(0)+\alpha_{1} y^{\prime}(0)=\alpha_{2}, \\
& N_{\tau}[y]=\beta_{0} y(\tau)+\beta_{1} y^{\prime}(\tau)=\beta_{2},
\end{aligned}
$$

where $a_{0}, a_{1}, a_{2} \in C[0, \tau], \alpha_{0}^{2}+\alpha_{1}^{2} \neq 0$, and $\beta_{0}^{2}+\beta_{1}^{2} \neq 0$. The purpose of this paper is to study closed form polynomial approximations to solutions of (1.1). In particular, we focus on a method which uses a uniform type norm as opposed to $L_{2}$ type norms used in other methods.

Let $P_{k}=\left\{p: p\right.$ is a polynomial of degree $k$ or less, $\left.N_{0}[p]=\alpha_{2}, N_{\tau}[p]=\beta_{2}\right\}$. We call $p_{k} \in P_{k}$ a minimax approximate solution (MAS) of (1.1) from $P_{k}$ if

Received November 22, 1977.

AMS (MOS) subject classifications (1970). Primary 65L10, $41 \mathrm{~A} 10$.

Key words and phrases. Minimax approximate solution, uniform approximation, boundary value problem.

* This author's research supported in part by a Faculty Research Grant at Marshall University. 


$$
\begin{aligned}
\max _{x \in[0, \tau]}\left|p_{k}^{\prime \prime}(x)-a_{0}(x) p_{k}^{\prime}(x)-a_{1}(x) p_{k}(x)-a_{2}(x)\right| \\
\quad=\inf _{p \in p_{k}} \max _{x \in[0, \tau]}\left|p^{\prime \prime}(x)-a_{0}(x) p^{\prime}(x)-a_{1}(x) p(x)-a_{2}(x)\right|
\end{aligned}
$$

Introducing the uniform norm $\|\cdot\|$ on $C[0, \tau]$ and the linear differential operator $D$ given by

$$
D[u]=u^{\prime \prime}-a_{0} u^{\prime}-a_{1} u
$$

the minimization problem (1.2) can be rewritten as

$$
\left\|D\left[p_{k}\right]-a_{2}\right\|=\inf _{p \in P_{k}}\left\|D[p]-a_{2}\right\| .
$$

Throughout we will assume that

$$
\text { the homogeneous boundary value problem } D[u]=0 \text {, }
$$

$$
N_{0}[u]=N_{\tau}[u]=0 \text { has no nontrivial solutions. }
$$

Condition (I) is equivalent to (1.1) having a unique solution $y \in C^{\prime}[0, \tau]$ (see [7]). In this paper, we consider a MAS as an approximation to $y$.

If (1.1) has homogeneous boundary conditions $\left(\alpha_{2}=\beta_{2}=0\right)$, a MAS can be viewed as a best approximation to $y$ from the linear subspace $P_{k}$ of $C_{0}^{\prime \prime}[0, \tau]=$ $\left\{u \in C^{\prime \prime}[0, \tau]: N_{0}[u]=N_{\tau}[u]=0\right\}$ with respect to the uniform type norm $\|\cdot\|_{D}$ on $C_{0}^{\prime \prime}[0, \tau]$ given by

$$
\|u\|_{D}=\|D[u]\| .
$$

This differs from the Rayleigh-Ritz approximates as the latter are best approximations to $y$ with respect to a similarly defined $L_{2}$ type norm (see [6]). In this light, a MAS differs from Galerkin or collocation approximates as a MAS results from a best uniform type norm approximation rather than an orthogonalization or a curve fitting process.

In the case of nonhomogeneous boundary conditions, a MAS results from a best approximation as follows: Fix $p^{*} \in P_{k}$ and let $P_{k}^{0}=\{q: q$ is a polynomial of degree $k$ or less, $\left.N_{0}[q]=N_{\tau}[q]=0\right\}$. Then any MAS $p_{k}$ can be written $p_{k}=q_{k}+p^{*}$, where

$$
\left\|D\left[q_{k}\right]-\left(a_{2}-D\left[p^{*}\right]\right)\right\|=\inf _{q \in P_{k}^{0}}\left\|D[q]-\left(a_{2}-D\left[p^{*}\right]\right)\right\| .
$$

Such a $q_{k}$ is a best approximation to $y-p^{*}$ from the linear subspace $P_{k}^{0}$ of $C_{0}^{\prime \prime}[0, \tau]$ with respect to the norm $\|\cdot\|_{D}$, or equivalently, $D\left[q_{k}\right]$ is a best uniform approximation to $a_{2}-D\left[p^{*}\right]$ from $D\left[P_{k}^{0}\right]=\left\{D[q]: q \in P_{k}^{0}\right\}$. Since $D\left[P_{k}^{0}\right]$ is a finite dimensional subspace of $C[0, \tau]$, such a $q_{k}$ exists and, thus, a MAS $p_{k}$ exists (see [2, p. 20]). However, $D\left[P_{k}^{0}\right]$ may not be a Haar subspace of $C[0, \tau]$, and, as a result, MAS's need not be unique.

For each $k \geqslant 3$, we select a MAS $p_{k}$ of (1.1) from $P_{k}$. In this paper, we show that $p_{k}, p_{k}^{\prime}$, and $p_{k}^{\prime \prime}$ converge uniformly to $y, y^{\prime}$, and $y^{\prime \prime}$, respectively. In fact, our analysis will yield corresponding orders of these convergences depending on smoothness 
properties of the coefficient functions $a_{0}, a_{1}$, and $a_{2}$ in (1.1). As computer computation of a MAS necessitates a discretization of the interval $[0, \tau]$, we analyze discrete minimax approximate solutions of (1.1) and show that they possess some rather pleasing convergence properties. This is followed by some examples of MAS's and comparison to actual solutions of (1.1). This work extends the ideas of [1], [3], [4] to boundary value problems.

2. Convergence and Orders of the Error. In this section, we show that if for each $k \geqslant 3$, a MAS $p_{k} \in P_{k}$ of (1.1) is fixed, then the sequences $\left\{p_{k}^{(i)}\right\}_{k=3}^{\infty}$ converge uniformly to $y^{(i)}, i=0,1,2$, where $y$ is the unique solution of (1.1). In addition, we estimate the orders of these convergences. It should be noted that condition (I) is assumed; and thus, a unique solution $y \in C^{\prime}[0, \tau]$ of (1.1) exists.

Let

$$
\delta_{k}=\inf _{p \in P_{k}}\left\|p^{\prime \prime}-a_{0} p^{\prime}-a_{1} p-a_{2}\right\|
$$

We shall require the following lemma.

Lemma 1. $\lim _{k \rightarrow \infty} \delta_{k}=0$.

Proof. Since $y, y^{\prime}, a_{0}, a_{1}, a_{2} \in C[0, \tau]$, the differential equation in (1.1) implies that $y^{\prime \prime} \in C[0, \tau]$. By the Weierstrass theorem, there is a sequence $\left\{r_{k}\right\}_{k=1}^{\infty}$ where $r_{k}$ is a polynomial of degree $k$ or less such that

$$
\lim _{k \rightarrow \infty}\left\|r_{k}-y^{\prime \prime}\right\|=0
$$

Let $H(x, \xi)$ be the Green's function for the boundary value problem $u^{\prime \prime}=0, u(0)=$ $u(\tau)=0$, and let $l(x)$ be the linear function such that $l(0)=y(0)$ and $l(\tau)=y(\tau)$. For reference on Green's functions, see [7]. Let the sequence $\left\{w_{k}\right\}_{k=1}^{\infty}$ be given by given by

$$
w_{k}(x)=l(x)+\int_{0}^{\tau} H(x, \xi) r_{k}(\xi) d \xi
$$

for $x \in[0, \tau]$. Then

$$
w_{k}^{\prime}(x)=l^{\prime}(x)+\int_{0}^{\tau} H_{x}(x, \xi) r_{k}(\xi) d \xi
$$

and $w_{k}^{\prime \prime}(x)=r_{k}(x)$ for $x \in[0, \tau]$. Thus, $w_{k}$ is a polynomial of degree $k+2$ or less. Furthermore,

$$
y(x)=l(x)+\int_{0}^{\tau} H(x, \xi) y^{\prime \prime}(\xi) d \xi
$$

and

$$
y^{\prime}(x)=l^{\prime}(x)+\int_{0}^{\tau} H_{x}(x, \xi) y^{\prime \prime}(\xi) d \xi
$$

for $x \in[0, \tau]$. Thus,

$$
\begin{gathered}
\left\|w_{k}-y\right\| \leqslant\left\|r_{k}-y^{\prime \prime}\right\| \tau^{2} / 8 \\
\left\|w_{k}^{\prime}-y^{\prime}\right\| \leqslant\left\|r_{k}-y^{\prime \prime}\right\| \tau / 2
\end{gathered}
$$


and

$$
\left\|w_{k}^{\prime \prime}-y^{\prime \prime}\right\|=\left\|r_{k}-y^{\prime \prime}\right\|
$$

By (2.2), (2.4), (2.5), and (2.6), $\lim _{k \rightarrow \infty}\left\|w_{k}^{(i)}-y^{(i)}\right\|=0, i=0,1,2$.

To complete the proof, we use the following observation. If

$$
s(x)=A x-\left(\frac{2 A+B}{\tau}\right) x^{2}+\left(\frac{A+B}{\tau^{2}}\right) x^{3},
$$

then $s(0)=s(\tau)=0, s^{\prime}(0)=A, s^{\prime}(\tau)=B$, and $\left\|s^{(i)}\right\| \rightarrow 0$ as $A \rightarrow 0$ and $B \rightarrow 0$, $i=0,1,2$.

From (2.3), $w_{k}(0)=y(0)$ and $w_{k}(\tau)=y(\tau)$, and from (2.5),

$$
\lim _{k \rightarrow \infty}\left|w_{k}^{\prime}(0)-y^{\prime}(0)\right|=\lim _{k \rightarrow \infty}\left|w_{k}^{\prime}(\tau)-y^{\prime}(\tau)\right|=0 .
$$

Let $s_{k}$ be the cubic polynomial in (2.7) with $s_{k}(0)=s_{k}(\tau)=0, s_{k}^{\prime}(0)=y^{\prime}(0)-w_{k}^{\prime}(0)$, and $s_{k}^{\prime}(\tau)=y^{\prime}(\tau)-w_{k}^{\prime}(\tau)$. Then $\lim _{k \rightarrow \infty}\left\|s_{k}^{(i)}\right\|=0, i=0,1,2$. Thus, $q_{k}=w_{k}+s_{k}$ $\in P_{k+2}$ and $\lim _{k \rightarrow \infty}\left\|q_{k}^{(i)}-y^{(i)}\right\|=0, i=0,1,2$.

Since $q_{k} \in P_{k+2}$,

$$
\begin{aligned}
0 \leqslant \delta_{k+2} & \leqslant\left\|q_{k}^{\prime \prime}-a_{0} q_{k}^{\prime}-a_{1} q_{k}-a_{2}\right\| \\
& \leqslant\left\|q_{k}^{\prime \prime}-y^{\prime \prime}\right\|+\left\|a_{0}\right\|\left\|q_{k}^{\prime}-y^{\prime}\right\|+\left\|a_{1}\right\|\left\|q_{k}-y\right\| .
\end{aligned}
$$

Thus, $\lim _{k \rightarrow \infty} \delta_{k}=0$ and Lemma 1 is proven.

Remark. In the proof of Lemma 1, (2.4), (2.5), and (2.6) indicate that $\left\|w_{k}^{(i)}-y_{k}^{(i)}\right\|=O\left(\left\|r_{k}-y^{\prime \prime}\right\|\right), i=0,1,2$. Moreover, (2.7) and the selection of $s_{k}$ imply that $\left\|s_{k}^{(i)}\right\|=O\left(\left\|r_{k}-y^{\prime \prime}\right\|\right), i=0,1,2$, and, hence, $\left\|q_{k}^{(i)}-y^{(i)}\right\|=O\left(\left\|r_{k}-y^{\prime \prime}\right\|\right)$. By (2.8), $\delta_{k}=O\left(\left\|r_{k}-y^{\prime \prime}\right\|\right)$.

Letting

$$
E_{k}\left(y^{\prime \prime}\right)=\inf \left\{\left\|p-y^{\prime \prime}\right\|: \quad p \text { is a polynomial of degree } k \text { or less }\right\},
$$

appropriate selection of $r_{k}$ implies that there is a constant $m_{1}$ independent of $k$ such that

$$
\delta_{k+2} \leqslant m_{1} E_{k}\left(y^{\prime \prime}\right)
$$

Before getting to the main result of this section, we rewrite (1.1) in its selfadjoint form. Let

$$
r(x)=\exp \left[-\int_{0}^{x} a_{0}(\xi) d \xi\right] \text { and } A(x)=r(x) a_{1}(x)
$$

for $x \in[0, \tau]$. Let $L$ be the linear differential operator given by $L[u]=\left(r u^{\prime}\right)^{\prime}-A u$. The selfadjoint form of the differential equation in (1.1) is

$$
L[y]=r a_{2} .
$$

Now condition (I) implies that a Green's function $G(x, \xi)$ for the boundary value problem $L[u]=0, N_{0}[u]=N_{\tau}[u]=0$ exists. If $f$ is the unique solution of the linear boundary value problem $L[u]=0, N_{0}[u]=\alpha_{2}, N_{\tau}[u]=\beta_{2}$, then the unique solution $w$ of the boundary value problem $L[w]=g, N_{0}[w]=\alpha_{2}, N_{\tau}[w]=\beta_{2}$, 
satisfies

$$
w(x)=f(x)+\int_{0}^{\tau} G(x, \xi) g(\xi) d \xi
$$

and

$$
w^{\prime}(x)=f^{\prime}(x)+\int_{0}^{\tau} G_{x}(x, \xi) g(\xi) d \xi
$$

for $x \in[0, \tau]$. Again see [7] for reference on Green's functions.

THEOREM 2. Assume conditon (I) holds and $y \in C^{\prime}[0, \tau]$ is the unique solution of (1.1). Suppose that for each $k \geqslant 3, a M A S p_{k}$ of (1.1) from $P_{k}$ is fixed. Then

$$
\lim _{k \rightarrow \infty}\left\|p_{k}^{(i)}-y^{(i)}\right\|=0, \quad i=0,1,2
$$

Proof. Let

$$
\Delta_{k}(x)=p_{k}^{\prime \prime}(x)-a_{0}(x) p_{k}^{\prime}(x)-a_{1}(x) p_{k}(x)-a_{2}(x)
$$

and $x \in[0, \tau]$. Note that $L[y]=r a_{2}$ and $L\left[p_{k}\right]=r\left(a_{2}+\Delta_{k}\right)$. Also, $y$ and $p_{k}$ satisfy the boundary conditions of (1.1). By (2.11) and (2.12),

and

$$
\begin{aligned}
\left\|p_{k}-y\right\| & \leqslant\left\|\Delta_{k}\right\| \sup _{x \in[0, \tau]} \int_{0}^{\tau}|G(x, \xi)| r(\xi) d \xi \\
& =\delta_{k} \sup _{x \in[0, \tau]} \int_{0}^{\tau}|G(x, \xi)| r(\xi) d \xi
\end{aligned}
$$

$$
\left\|p_{k}^{\prime}-y^{\prime}\right\| \leqslant \delta_{k} \sup _{x \in[0, \tau]} \int_{0}^{\tau}\left|G_{x}(x, \xi)\right| r(\xi) d \xi .
$$

From the differential equation of (1.1) and (2.13),

$$
\begin{aligned}
\left\|p_{k}^{\prime \prime}-y^{\prime \prime}\right\| \leqslant \delta_{k}+\left\|a_{0}\right\|\left\|p_{k}^{\prime}-y^{\prime}\right\|+\left\|a_{1}\right\|\left\|p_{k}-y_{k}\right\| \\
\leqslant \delta_{k}\left(1+\left\|a_{0}\right\| \sup _{x \in[0, \tau]} \int_{0}^{\tau}|G(x, \xi)| r(\xi) d \xi\right. \\
\left.+\left\|a_{1}\right\| \sup _{x \in[0, \tau]} \int_{0}^{\tau}\left|G_{x}(x, \xi)\right| r(\xi) d \xi\right) .
\end{aligned}
$$

Now by Lemma 1, (2.14), (2.15) and (2.16), $\lim _{k \rightarrow \infty}\left\|p_{k}^{(i)}-y^{(i)}\right\|=0, i=0,1,2$. Thus, Theorem 2 is proven.

Remark. From (2.9), (2.14), (2.15), and (2.16), we see that $\left\|p_{k}^{(i)}-y^{(i)}\right\|=$ $O\left(E_{k-2}\left(y^{\prime \prime}\right)\right), i=0,1,2$, and thus there is a constant $m_{2}$ independent of $k$ such that

$$
\left\|p_{k}^{(i)}-y^{(i)}\right\| \leqslant m_{2} E_{k-2}\left(y^{\prime \prime}\right) \text {. }
$$

By Jackson's theorem [2, p. 147], if $y^{\prime \prime} \in C^{n}[0, \tau]$, then $E_{k-2}\left(y^{\prime \prime}\right)=O\left(1 / k^{n}\right)$. From the differential equation of (1.1), $y^{\prime \prime} \in C^{n}[0, \tau]$ whenever $a_{0}, a_{1}, a_{2} \in C^{n}[0, \tau]$. Thus, we have the following corollary to the proof of Theorem 2 .

COROllary 3. If $a_{0}, a_{1}, a_{2} \in C^{n}[0, \tau]$, then there is a constant $m$ independent of $k$ such that

$$
\left\|p_{k}^{(i)}-y^{(i)}\right\| \leqslant m / k^{n}, \quad i=0,1,2
$$


3. Discretization. In practice, rather than finding a $p_{k} \in P_{k}$ satisfying (1.2), one would find a $p_{k, X} \in P_{k}$ such that

$$
\begin{aligned}
& \max _{x \in X}\left|p_{k, X}^{\prime \prime}(x)-a_{0}(x) p_{k, X}^{\prime}(x)-a_{1}(x) p_{k, X}(x)-a_{2}(x)\right| \\
& =\inf _{p \in P_{k}} \max _{x \in X}\left|p^{\prime \prime}(x)-a_{0}(x) p^{\prime}(x)-a_{1}(x) p(x)-a_{2}(x)\right|,
\end{aligned}
$$

where $X$ is a closed subset (usually finite) of $[0, \tau]$. We call such a $p_{k} \in P_{k}$ a discrete minimax approximate solution of (1.1) from $P_{k}$. In this section, we show that if $X$ is "sufficiently dense" in $[0, \tau]$, then any discrete MAS is nearly a MAS.

The density of $X$ in $[0, \tau]$ is defined to be

and in contrast to (2.1) let

$$
d(X)=\sup _{s \in[0, \tau]} \inf _{t \in X}|s-t|
$$

$$
\delta_{k, X}=\inf _{p \in p_{k}} \max _{x \in X}\left|p^{\prime \prime}(x)-a_{0}(x) p^{\prime}(x)-a_{1}(x) p_{k}(x)-a_{2}(x)\right| .
$$

THEOREM 4. Given $\epsilon>0$, there is a $\delta>0$ such that if $d(X)<\delta$, then $0 \leqslant \delta_{k}-$ $\delta_{k, X}<\epsilon$.

Proof. Let $\epsilon>0$ be given. Let $0<\epsilon_{1}<\epsilon / \delta_{k}$. Applying Lemma 1 on p. 85 of [2] to the finite dimensional linear span of $\left\{p^{\prime \prime}-a_{0} p^{\prime}-a_{1} p-a_{2}: p \in P_{k}\right\}$, there is a $\delta>0$ independent of $X$ such that if $d(X)<\delta$, then

$$
\begin{aligned}
\max _{x \in[0, \tau]}\left|p^{\prime \prime}(x)-a_{0}(x) p^{\prime}(x)-a_{1}(x) p(x)-a_{2}(x)\right| \\
\leqslant\left(1+\epsilon_{1}\right) \max _{x \in X}\left|p^{\prime \prime}(x)-a_{0}(x) p^{\prime}(x)-a_{1}(x) p(x)-a_{2}(x)\right|
\end{aligned}
$$

for all $p \in P_{k}$. It is clear that $\delta_{k, X} \leqslant \delta_{k}$. Let $p_{k, X}$ be a discrete MAS of (1.1) from $P_{k}$ corresponding to a closed subset $X$ of $[0, \tau]$ with $d(X)<\delta$. Then

$$
\begin{aligned}
\delta_{k} & \leqslant \max _{x \in[0, \tau]}\left|p_{k, X}^{\prime \prime}(x)-a_{0}(x) p_{k, X}^{\prime}(x)-a_{1}(x) p_{k, X}(x)-a_{2}(x)\right| \\
& \leqslant\left(1+\epsilon_{1}\right) \max _{x \in X}\left|p_{k, X}^{\prime \prime}(x)-a_{0}(x) p_{k, X}^{\prime}(x)-a_{1}(x) p_{k, X}(x)-a_{2}(x)\right| \\
& =\left(1+\epsilon_{1}\right) \delta_{k, X}<\delta_{k, X}+\epsilon .
\end{aligned}
$$

The proof of Theorem 4 is complete.

The following corollary to the proof of Theorem 4 indicates that any discrete MAS is nearly a MAS whenever $X$ is sufficiently dense in $[0, \tau]$. The proof is contained in (3.3).

Corollary 5. Given $\epsilon>0$ there is $a \delta>0$ such that if $d(X)<\delta$, then

$$
\begin{aligned}
\delta_{k} & \leqslant \max _{x \in[0, \tau]}\left|p_{k, X}^{\prime \prime}(x)-a_{0}(x) p_{k, X}^{\prime}(x)-a_{1}(x) p_{k, X}(x)-a_{2}(x)\right| \\
& <\delta_{k}+\epsilon
\end{aligned}
$$

where $p_{k, X}$ satisfies (3.1). 
In view of the proof of Theorem 2, we see from Corollary 5, that when $X$ is sufficiently dense in $[0, \tau]$, a corresponding discrete MAS approximates the solution of (1.1) nearly as well as a MAS.

The last result of this section concentrates on how well a discrete MAS approximates a MAS. For any closed subset $X$ of $[0, \tau]$, let $p_{k, X} \in P_{k}$ satisfy (3.1) and define

$$
\Delta_{k, X}(x)=p_{k, X}^{\prime \prime}(x)-a_{0}(x) p_{k, X}^{\prime}(x)-a_{1}(x) p_{k, X}(x)-a_{2}(x)
$$

Then $p_{k, X}$ is a solution of the boundary value problem $L[u]=r\left(a_{2}+\Delta_{k, X}\right), N_{0}[u]=$ $\alpha_{2}, N_{\tau}[u]=\beta_{2}$. Then by (2.11),

$$
p_{k, X}(x)=f(x)+\int_{0}^{\tau} G(x, \xi) r(\xi)\left(a_{2}(\xi)+\Delta_{k, X}(\xi)\right) d \xi
$$

for $x \in[0, \tau]$. For $X$ sufficiently dense in $[0, \tau]$, Corollary 5 insures that

$$
\left\|p_{k, X}\right\| \leqslant\|y\|+\left(\delta_{k}+1\right) \sup _{x \in[0, \tau]} \int_{0}^{\tau}|G(x, \xi)| r(\xi) d \xi .
$$

Now consider a sequence $\left\{X_{n}\right\}_{n=1}^{\infty}$ of closed subsets of $[0, \tau]$, where $d\left(X_{n}\right) \rightarrow 0$ as $n \rightarrow \infty$. By (3.4), the sequence $\left\{p_{k, X_{n}}\right\}_{n=1}^{\infty}$ is uniformly bounded over $[0, \tau]$ and, thus, has a cluster point $\bar{p}_{k} \in P_{k}$.

THEOREM 6. The polynomial $\bar{p}_{k}$ is a MAS of (1.1) from $P_{k}$.

Proof. Suppose $p_{k, X_{n(l)}} \rightarrow \bar{p}_{k}$. By Corollary 5,

$$
\lim _{l \rightarrow \infty}\left\|p_{k, X_{n(l)}^{\prime \prime}}^{\prime \prime}-a_{0} p_{k, X_{n(l)}^{\prime}}^{\prime}-a_{1} p_{k, X_{n(l)}}-a_{2}\right\|=\delta_{k} \text {. }
$$

But $p_{k, X_{n(l)}^{(i)}} \rightarrow \bar{p}_{k}^{(i)}, i=0,1,2$. Thus

$$
\begin{gathered}
\lim _{l \rightarrow \infty}\left\|p_{k, X_{n(l)}}^{\prime \prime}-a_{0} p_{k, X_{n(l)}^{\prime}}^{\prime}-a_{1} p_{k, X_{n(l)}}-a_{2}\right\| \\
=\left\|\bar{p}_{k}^{\prime \prime}-a_{0} \bar{p}_{k}^{\prime}-a_{1} \bar{p}_{k}-a_{2}\right\| .
\end{gathered}
$$

Thus, $\bar{p}_{k}$ satisfies (1.2).

4. Examples. In this section, we report the results of five computer examples of minimax approximate solutions of boundary value problems. Two algorithms have been employed. In Examples 1, 2, 4, and 5, the first two coefficients of a MAS can be expressed in terms of the remaining coefficients, and finding a MAS becomes a linear best uniform approximation problem. In these cases, the second algorithm of Remes [2] was used. In the discrete case, a MAS can be expressed as a solution of a linear programming problem. The linear programming algorithm was used in all cases with the interval discretized with 21 evenly spaced points. In Examples 1, 2, 4, and 5, there are only slight differences in the MAS's and in the maximum errors computed over 21 evenly spaced points in the interval using both algorithms. In these examples, the Remes algorithm provided somewhat smaller errors, and we report these results. In Example 3, the first two coefficients of a MAS cannot be expressed in terms of the remaining coefficients, and it is not expedient to use the Remes algorithm. The results 
for this example come from the linear programming algorithm. All computations were done on an IBM 370 computer in double precision arithmetic. For simplicity of notation, we shall denote the discrete MAS's by $p_{k}$.

Example 1.

$$
y^{\prime \prime}+\pi^{2} y=0, \quad x \in[0,1], \quad y(0)=0, \quad y^{\prime}(1)=-\pi .
$$

For $k=7$,

$$
\begin{aligned}
p_{7}(x)= & 3.14159 x-0.00284 x^{2}-5.09959 x^{3} \\
& -0.39503 x^{4}+3.53380 x^{5}-1.17794 x^{6}-0.0000008 x^{7}
\end{aligned}
$$

with $\delta_{7}=0.0057$. The actual solution is $y(x)=\sin \pi x$ and the maximum error is

$$
\max _{x \in[0,1]}\left|p_{7}(x)-\sin \pi x\right|=0.000053
$$

Example 2.

$$
\begin{array}{ll}
y^{\prime \prime}=2 x y^{\prime}+2 y, & x \in[0,1], \\
y(0)-y^{\prime}(0)=1, & 2 y(1)-y^{\prime}(1)=0 .
\end{array}
$$

A MAS degree of 6 is

$$
\begin{aligned}
p_{6}(x)= & 1.00022+0.00023 x+1.01817 x^{2} \\
& -0.25924 x^{3}+1.38320 x^{4}-1.19479 x^{5}+0.76947 x^{6}
\end{aligned}
$$

with $\delta_{6}=0.036$. The actual solution is $y(x)=\exp \left(x^{2}\right)$ and

$$
\max _{x \in[0,1]}\left|p_{6}(x)-\exp \left(x^{2}\right)\right|=0.00093
$$

Example 3.

$$
\begin{aligned}
& y^{\prime \prime}=\frac{-1}{1+x} y^{\prime}+x y-x \ln (1+x), \quad x \in[0,1], \\
& y(0)+y^{\prime}(0)=1 \\
& y(1)=\ln (2)=0.69147 .
\end{aligned}
$$

A MAS of degree 6 is

$$
\begin{aligned}
p_{6}(x)= & 0.00005+0.99995 x-0.49995 x^{2} \\
& +0.32390 x^{3}-0.20309 x^{4}+0.09135 x^{5}-0.01951 x^{6}
\end{aligned}
$$

with $\delta_{6}=0.00093$. The actual solution is $y(x)=\ln (1+x)$ with a uniform error

$$
\max _{x \in[0,1]}\left|p_{6}(x)-\ln (1+x)\right|=0.00005
$$

Example 4.

$$
\begin{aligned}
& y^{\prime \prime}=-\pi^{2} y \sin ^{2} x-\pi^{2} \sin \pi x \cos ^{2} x, \quad x \in[0,1], \\
& y(0)=y(1)=0 .
\end{aligned}
$$


For $k=7$,

$$
\begin{aligned}
p_{7}(x)= & 3.14151 x-0.00292 x^{2}-5.09855 x^{3} \\
& -0.39681 x^{4}+3.53450 x^{5}-1.17731 x^{6}-0.00042 x^{7}
\end{aligned}
$$

with $\delta_{7}=0.0058$. The actual solution is $y(x)=\sin \pi x$ and

$$
\max _{x \in[0,1]}\left|p_{7}(x)-\sin \pi x\right|=0.000042
$$

Examples 1-4 indicate that for relatively low degrees MAS's provide good approximations to the solutions of the boundary value problems. It is interesting to note that in each of the examples above the maximum error is one or two orders of magnitude smaller than the corresponding $\delta_{k}$. In our last example, we consider boundary value problems which "nearly" do not satisfy condition (I).

Example 5.

$$
y^{\prime \prime}+\pi^{2} y=0, \quad x \in[0, \tau], \quad y(0)=0, \quad y(\tau)=1 .
$$

For $\tau=1$, the boundary value problem does not satisfy condition (I) and, in fact, does not have a solution. For $0<\tau<1$, condition (I) is satisfied and the solution is

$$
y(x)=\frac{\sin \pi x}{\sin \pi \tau}
$$

For $\tau=.99$, a MAS of degree 7 is

$$
\begin{aligned}
p_{7}(x)= & 100.02520 x-0.08527 x^{2}-162.46945 x^{3} \\
& -12.08374 x^{4}+111.55158 x^{5}-36.67208 x^{6}-0.26634 x^{7}
\end{aligned}
$$

with $\delta_{7}=0.170$. The maximum error is 0.00355 , and the relative error is $0.011 \%$.

For $\tau=.999$ and $k=7$,

$$
\begin{aligned}
p_{7}(x)= & 1001.14814 x-0.89931 x^{2}-1625.22006 x^{3} \\
& -125.38559 x^{4}+1125.16506 x^{5}-374.54331 x^{6}-0.26606 x^{7}
\end{aligned}
$$

with $\delta_{7}=1.80$. The uniform error is 0.352 with a relative error of $0.11 \%$.

In either case, the maximum error is not as dramatically small as in Examples $1-4$; however, the relative errors are quite small.

We conclude this section with some remarks of the two algorithms used. Although the Remes algorithm is not guaranteed to work in the absence of the Haar condition, no failures were encountered with this algorithm. In Examples 1, 2, 4 and 5, the Remes algorithm' produced virtually the same results as those of the linear programming algorithms. The Remes algorithm appears to be considerably faster than the linear programming algorithm. In Examples 1, 2, 4, and 5, the Remes algorithm required between 3 and 4 seconds of computer processing unit (CPU) time and the linear programming algorithm required between 8 and 10 seconds of CPU times.

5. Conclusions. Approximation theory techniques have been used to provide approximate solutions of boundary value problems. Convergence is guaranteed under rather general conditions and appropriate orders on the uniform error are obtained. 
Although it has been shown that discretization does not significantly alter the approximation properties of a MAS, further research needs to be done with algorithms for finding MAS's. Particularly, it would be interesting to characterize those problems in which the Remes algorithm applies.

Department of Mathematics

Marshall University

Huntington, West Virginia 25701

Department of Mathematics

College of Charleston

Charleston, South Carolina 29401

1. G. ALLINGER \& M. S. HENRY, "Approximate solutions of differential equations with deviating arguments," SIAM J. Numer. Anal., v. 13, 1976, pp. 412-426.

2. E. W. CHENEY, Introduction to Approximation Theory, McGraw-Hill, New York, 1966.

3. M. S. HENRY, "Best approximate solutions of nonlinear differential equations," $J$. Approximation Theory, v. 3, 1970, pp. 59-65.

4. M. S. HENRY \& K. L. WIGGINS, "Applications of approximation theory to the initial value problem," J. Approximation Theory, v. 17, 1976, pp. 66-85.

5. A. G. PETSOULAS, "The approximate solution of Volterra integral equations," $J$. Approximation Theory, v. 14, 1975, pp. 152-159.

6. P. M. PRENTER, Splines and Variational Mathods, Wiley, New York, 1975.

7. H. SAGAN, Boundary and Eigenvalue Problems in Mathematical Physics, Wiley, New York, 1961. 